政

\title{
Obesidade: fatores de risco para doença cardiovascular na infância
}

\author{
Mateus G. Tavares, ${ }^{1 *}$ Isabel R. Madeira, ${ }^{2}$ Fernanda M. Gazolla, ${ }^{3}$ Cecilia N. M. Carvalho, ${ }^{4}$ Cecilia L. Oliveira, ${ }^{4}$ \\ Marcos A. Borges, ${ }^{3}$ Paulo F. Collett-Solberg, ${ }^{5}$ Cláudia B. Monteiro, ${ }^{6}$ Ana P. Bordallo, ${ }^{3}$ Clarice B. Medeiros, ${ }^{3}$ \\ Daniel S. Gilban ${ }^{3}$
}

\section{Resumo}

Introdução: Diante do aumento da prevalência da obesidade em crianças, o objetivo do estudo foi comparar crianças impúberes com obesidade e obesidade grave em relação aos fatores de risco para doenças cardiovasculares e diabetes mellitus do tipo 2.

Materiais e Métodos: Foi feito um estudo transversal com 84 crianças que comparou 52 crianças obesas com 32 obesas graves, oriundas do Ambulatório de Pesquisa em Obesidade Infantil do Hospital Universitário Pedro Ernesto, quanto às médias de circunferência da cintura, glicose, lipídios séricos, insulina, homeostasis model assessment for insulin resistance (HOMA-IR), pressão arterial sistólica (PAS) e pressão arterial diastólica (PAD), adiponectina e leptina. Compararam-se as frequências de acantose nigricans, síndrome metabólica e aumento de alterações de cintura, pressão arterial, glicose, lipídios séricos e insulina.

Resultados: Houve diferenças estatisticamente significativas quando se compararam as médias dos grupos estudados quanto à circunferência da cintura, PAS, PAD, insulina e HOMA-IR. $\mathrm{O}$ mesmo ocorreu em relação às frequências de acantose nigricans e alteração de cintura e insulina.

Discussão: Apesar de o estudo ter sido realizado com crianças em idade tenra, achados de fatores de risco para doença cardiovascular aterosclerótica em ambos os grupos são preocupantes. Além disso, a diferença substancial nos resultados desses grupos demonstra que a obesidade grave já apresenta consequências mais significativas, até mesmo na idade tenra.

Descritores: Criança; Obesidade; Fatores de risco; Diabetes mellitus tipo 2; Doenças cardiovasculares.

\section{Abstract \\ Obesity: Cardiovacular risk factors in childhood}

Introduction: Given the increasing prevalence of obesity in children, the purpose of this study is to compare prepubescent children with obesity and severe obesity concerning risk factors for cardiovascular diseases and type 2 diabetes mellitus.

Materials and Methods: A cross-sectional study of 84 children was done, comparing 52 obese children with 32 children with severe obesity, concerning their average waist circumference, glucose, serum lipids, insulin, homeostasis model assessment for insulin resistance(HOMA-IR), systolic blood pressure (SBP) and diastolic blood pressure (DBP), adiponectin and leptin. The frequencies of acanthosis nigricans, metabolic syndrome and altered waist, blood pressure, glucose, serum lipids and insulin were compared. Results: There was a substantial difference when comparing
1. Faculdade de Ciências Médicas. Universidade do Estado do Rio de Janeiro. Rio de Janeiro, RJ, Brasil.

2 Departamento de Pediatria. Faculdade de Ciências Médicas. Universidade do Estado do Rio de Janeiro. Rio de Janeiro, RJ, Brasil.

3 Unidade Docente Assistencial de Endocrinologia e Metabologia. Hospital Universitário Pedro Ernesto. Universidade do Estado do Rio de Janeiro. Rio de Janeiro, RJ, Brasil.

4 Departamento de Nutrição Aplicada. Instituto de Nutrição. Universidade do Estado do Rio de Janeiro. Rio de Janeiro, RJ, Brasil.

5 Departamento de Medicina Interna. Faculdade de Ciências Médicas. Universidade do Estado do Rio de Janeiro. Rio de Janeiro, RJ, Brasil.

6. Núcleo de Estudos da Saúde do Adolescente (NESA). Hospital Universitário Pedro Ernesto. Universidade do Estado do Rio de Janeiro. Rio de Janeiro, RJ, Brasil

\section{*Endereço para correspondência: \\ Rua Governador Martim de Sá, 340, casa A, \\ Rio de Janeiro, RJ, Brasil. CEP: 21910-062. \\ E-mail: mateus.garcia.tavares@gmail.com}

Revista HUPE, Rio de Janeiro, 2016;15(2):99-105

doi: 10.12957/rhupe.2016.28233

Recebido em 28/07/2016. Aprovado em 18/10/2016.

the averages of the groups concerning the following parameters: waist circumference, SBP, DBP, insulin, and HOMA-IR. A substantial difference was also detected in the frequency of acanthosis nigricans, alteration of waist and of insulin.

Discussion: Although the study was conducted with children at an early age, findings of atherosclerotic cardiovascular risk factors in both groups are worrisome. In addition, the substantial difference in the results in both groups shows that severe obesity has more significant consequences than obesity, even at early ages..

Keywords: Child; Obesity; Risk factors; Diabetes mellitus, Type 2; Cardiovascular disease.

\section{Resumen}

\section{Obesidad: factores de riesgo cardiovascular en la infancia}

Introducción: Dada la creciente prevalencia de la obesidad en niños, el objetivo del estudio fue comparar niños impúberes con obesidad y niños con obesidad severa en relación con los factores de riesgo para enfermedades cardiovasculares y 


\section{Artigo original}

diabetes mellitus tipo 2 .

Materiales y Métodos: Se hizo un estudio transversal con 84 niños que comparó 52 niños obesos con 32 obesos severos, provenientes del Ambulatorio de Investigación en Obesidad Infantil del Hospital Universitario Pedro Ernesto, en relación con la circunferencia media de la cintura, glucosa, lípidos séricos, insulina, índice de resistencia a la insulina (homeostasis model assessment for insulin resistance/ HOMA-IR), presión arterial sistólica (PAS), presión arterial diastólica (PAD), adiponectina y leptina. Además, se comparó la frecuencia de la acantosis nigricans, del síndrome metabólico y del aumento de las alteraciones de la cintura, de la presión arterial, de la glucosa, de los lípidos séricos y de la insulina.

Resultados: Se observaron diferencias estadísticamente signi-

\section{Introdução}

O excesso de peso representa um grave problema de saúde pública, afetando tanto países desenvolvidos quanto em desenvolvimento. No mundo, a proporção de adultos com um índice de massa corporal (IMC) <= $25 \mathrm{~kg} / \mathrm{m}^{2}$ aumentou de $28,8 \%$ para $36,9 \%$ em homens e de $29,8 \%$ para $38,0 \%$ em mulheres, entre 1980 e $2013 .{ }^{1}$ No Brasil, a obesidade, inclusive em crianças, também vem se tornando bastante prevalente. A prevalência de obesidade em crianças de 5 a 9 anos, por exemplo, aumentou de $2,9 \%$ para $16,6 \%$ no sexo masculino e de $1,8 \%$ para $11,8 \%$ no sexo feminino, no período de 1974-1975 a 2008-2009.² Além disso, a obesidade entre crianças e adolescentes é, atualmente, um dos principais problemas de saúde pública, não só por causa da maior possibilidade de ter obesidade na idade adulta, ${ }^{3}$ mas também por causa do aumento do risco de aparecimento, mesmo precoce, dos outros componentes da síndrome metabólica. ${ }^{4}$

A obesidade se associa a outras comorbidades na chamada síndrome metabólica (SM). Esta se caracteriza, em adultos, pela associação de pelo menos três dos seguintes fatores de risco: obesidade abdominal, hipertensão arterial sistêmica, hipertrigliceridemia, glicemia elevada em jejum e baixos níveis de lipoproteína de alta densidade (HDL-colesterol). Tal associação é considerada como sendo de risco aumentado para diabetes mellitus tipo 2 (DM2) e doença cardiovascular aterosclerótica. ${ }^{5}$ Para crianças, já existe uma definição de SM. O International Diabetes Federation (IDF) propôs, para crianças maiores de 10 anos, uma definição de SM baseada na presença de circunferência da cintura aumentada mais pelo menos dois elementos dos que se seguem: hipertrigliceridemia; HDL-colesterol baixo; hipertensão arterial sistêmica; e glicemia de jejum ficativas al comparar las medias de los grupos estudiados con respecto a la circunferencia de la cintura PAS, PAD, insulina y HOMA-IR. Lo mismo ocurrió en relación con la frecuencia de la acantosis nigricans y las alteraciones de la cintura y la insulina.

Discusión: A pesar de que el estudio se llevó a cabo con niños en edad temprana, la existencia de factores de riesgo para enfermedad cardiovascular aterosclerótica en ambos grupos es preocupante. Además, la diferencia sustancial en los resultados de ambos grupos demuestra que la obesidad severa presenta consecuencias más significativas incluso en la edad temprana.

Palabras clave: Niño; Obesidad; Factores de Riesgo; Diabetes mellitus tipo 2; Enfermedades cardiovasculares.

alterada ou DM2. ${ }^{5}$

A fisiopatologia da obesidade envolve desequilíbrio energético entre ingestão e gasto. Vários fatores neuroendócrinos implicam nesse desequilíbrio, como as adipocitoquinas, proteínas produzidas pelo tecido adiposo. Exemplos dessas adipocitoquinas são a leptina e a adiponectina, que estão envolvidas na regulação do metabolismo dos carboidratos e gorduras. ${ }^{6}$

Essa fisiopatologia também envolve a insulina. Trata-se de um hormônio com importantes funções metabólicas, tais como, promover adequada captação periférica de glicose, suprimir a gliconeogênese hepática e inibir a produção de lipoproteína de muito baixa densidade. A resistência insulínica é definida como a ineficiência da insulina plasmática, em concentrações normais, para exercer tais funções. Na faixa etária pediátrica, a obesidade parece ser importante gatilho para a resistência insulínica, o que torna as crianças obesas um grupo de risco, visto que a resistência insulínica tem papel fisiopatológico importante na gênese da doença cardiovascular aterosclerótica. ${ }^{7}$ Essa resistência pode ser avaliada por meio do índice chamado, homeostasis model assessment for insulin resistance (HOMA-IR), calculado multiplicando-se a glicemia (mmol/L) pela insulina $(\mu \mathrm{UI} / \mathrm{mL})$, ambas em jejum, e dividindo-se por $22,5 .{ }^{8} \mathrm{~A}$ resistência também pode ser avaliada pela presença de acantose nigricans, considerado seu marcador cutâneo. ${ }^{9}$

Outro ponto preocupante é o crescente aumento de obesidade grave entre crianças e adolescentes. A obesidade grave afeta entre $4 \%$ e $6 \%$ de todos os jovens nos Estados Unidos. ${ }^{10}$ Além disso, dados sugerem que, em comparação com as crianças com sobrepeso ou com obesidade, as crianças com obesidade grave têm um perfil de risco cardiometabólico mais adverso e demonstraram os primeiros sinais de disfunção vascular e 
aterosclerose subclínica. ${ }^{11}$ Além disso, o elevado IMC na infância está associado com o aumento do risco de DM2 e doença cardiovascular na idade adulta, e até mesmo morte em idades mais prematuras. ${ }^{12,13}$ Desse modo, são crescentes os questionamentos no âmbito científico acerca da necessidade de maior atenção às crianças com obesidade grave e sobre o fato de crianças obesas não classificadas como graves terem menores riscos.

Diante dessa perspectiva, o objetivo desse trabalho foi comparar grupos de crianças impúberes obesas com aquelas também impúberes, mas que possuem obesidade grave, em relação aos fatores de risco para doença cardiovascular aterosclerótica, insulina, leptina e adiponectina.

\section{Métodos}

Trata-se de um estudo de observação, transversal, realizado no Ambulatório de Pesquisa em Obesidade Infantil do Hospital Universitário Pedro Ernesto da Universidade do Estado do Rio de Janeiro, com crianças oriundas do Ambulatório de Pediatria Geral do mesmo hospital. Foram estudadas crianças com obesidade e com obesidade grave, com idades entre 5 e 11 anos, impúberes segundo o critério de Tanner. ${ }^{14} \mathrm{~A}$ partir dos dados obtidos através da avaliação nutricional, calculou-se o indicador IMC considerando-se a razão peso atual $(\mathrm{Kg})$ e o quadrado da estatura $\left(\mathrm{m}^{2}\right)$ sendo este índice avaliado com o auxílio dos referenciais da Organização Mundial da Saúde ${ }^{15}$ e interpretado utilizando os pontos de corte preconizados por esta de acordo com o gênero e a faixa etária, sendo considerada: eutrofia maior ou igual escore $Z-2$ e menor que o escore $Z+1$; sobrepeso - maior ou igual escore $Z+1$ e menor ou igual escore $Z+2$; obesidade - maior que o escore $Z+2$ e menor ou igual ao escore $Z+3$; e obesidade grave - maior que o escore $Z+3 .^{15}$

As crianças eram saudáveis sob outros aspectos, não estavam incluídas em qualquer programa para perda de peso e foram selecionadas por ordem de admissão ao Ambulatório de Pesquisa em Obesidade Infantil do Hospital Universitário Pedro Ernesto da Universidade do Estado do Rio de Janeiro. O tamanho amostral utilizado foi de 84 crianças, sendo 52 crianças participantes do grupo de obesidade e 32 participantes do grupo de obesidade grave. Essa amostra foi suficiente para um poder estatístico de $80 \%$, com nível de significância de $5 \%$, para um erro de $5 \%$, tomando-se como base a população de crianças atendidas no ambulatório de pediatria geral ${ }^{16} \mathrm{As}$ crianças recrutadas para o ambulatório de pesquisa foram atendidas com data marcada. O res- ponsável legal foi informado e esclarecido previamente à realização de qualquer procedimento e assinou um termo de consentimento livre e esclarecido. Naquela data, todas as crianças foram submetidas à avaliação clínica completa, segundo um protocolo que incluiu avaliação visual de acantose nigricans em pescoço, axilas, falanges, cotovelos e joelhos. ${ }^{17}$

A pesagem foi sem sapatos e com roupas leves, em balança da marca Filizola ${ }^{\circledR}$ (Filizola, São Paulo,SP, Brasil). As estaturas foram medidas sem sapatos, em estadiômetro de parede do tipo Harpenden-Holtain, da marca Tonelli ${ }^{\circledR}$ (Tonelli, Criciúma, SC, Brasil). A medida da circunferência da cintura foi tomada a uma altura logo acima da borda lateral mais alta do ilíaco, ao final de uma expiração normal, segundo recomendações da Third National Health and Nutrition Examination Survey (NHANES III) estadunidense, ${ }^{18} \mathrm{com}$ fita antropométrica milimetrada, da marca Mabbis ${ }^{\circledR}$, modelo Gulik (Cardiomed, Curitiba, PR, Brasil). Além disso, a circunferência da cintura foi definida como aumentada, quando a medida estava dentro ou acima do percentil 90 para o sexo e idade na tabela de NHANES III ${ }^{18}$ que combina as crianças de etnia africana-americana, europeia-americana e mexicana-americana. A medida da pressão arterial foi tomada no braço direito, com a criança sentada, em repouso, com esfignomanômetro da marca $T y \cos { }^{\circledR}$ (Welch Allyn Company, Arden, DE, EUA), usando-se manguitos de tamanhos adequados. Para definição de pressão arterial aumentada, adotou-se o critério recomendado pela I Diretriz de Prevenção da Aterosclerose na Infância e na Adolescência da Sociedade Brasileira de Cardiologia. ${ }^{19}$

Os pontos de corte adotados para glicemia, colesterol, HDL-colesterol, LDL-colesterol, triglicerídeos e insulina, todos em jejum, foram os recomendados na I Diretriz de Prevenção da Aterosclerose na Infância e na Adolescência da Sociedade Brasileira de Cardiologia: glicemia aumentada, valores variando de 100 a 125 $\mathrm{mg} / \mathrm{dL}$; colesterol alterado, valores a partir de $150 \mathrm{mg} /$ dL, sendo que valores de 150 a 169 são considerados limítrofes e maior ou igual a 170, aumentados; LDL-colesterol alterado, valores a partir de $100 \mathrm{mg} / \mathrm{dL}$, sendo que valores de 100 a 129 são considerados limítrofes e maior ou igual a 130, aumentados; insulina aumentada, valores a partir de $15 \mu \mathrm{IU} / \mathrm{mL}^{19}$

O índice HOMA-IR foi calculado multiplicando-se a glicose de jejum (mmol/L) pela insulina de jejum ( $\mu \mathrm{IU} /$ $\mathrm{mL}$ ) e dividindo-se por $22,5 .^{8}$

A classificação empregada para SM foi adaptada do IDF para crianças acima de 10 anos - circunferência 


\section{Artigo original}

aumentada, e, pelo menos, mais dois dos seguintes critérios: glicemia de jejum alterada ou DM2; HDL-colesterol baixo; triglicerídeos altos; hipertensão arterial sistêmica. ${ }^{5}$ Os últimos três critérios seguiram os valores mencionados nos parágrafos anteriores.

A coleta de sangue para os exames laboratoriais foi feita em um dia próximo, em momento posterior à avaliação clínica, após jejum de 12 horas. Os exames realizados foram dosagem sérica de glicose, colesterol total e HDL-colesterol, triglicerídeos, insulina, leptina e adiponectina. Os quatro primeiros foram medidos em um analisador Konelab com o kit BT Winer, que emprega os seguintes métodos de ensaio: para a glicose, o método enzimático GOD-PAP (oxidase); para o colesterol, o método enzimático CHOP-POD (esterase-oxidase); para as triglicérides, o método enzimático GPO-PAP (oxidase); e para o colesterol HDL, o método colorimétrico enzimático sem precipitação (Winterlab, Rosario, Santa Fe, Argentina). O LDL-colesterol foi calculado pela fórmula de Friedwald: LDL-colesterol = colesterol total - (HDL-colesterol + triglicérides $/ 5){ }^{20}$

A insulina foi dosada no laboratório de endocrinologia do hospital do estudo, no equipamento GAMA $\mathrm{C} 12$, por meio de um kit que utiliza o método Coat-A-Count, um radioimunoensaio fase sólida marcado com 125 I (DPC, Los Angeles, CA, EUA). Os coeficientes de variação intraensaio e interensaio foram 3,1-9,3\% e 4,9-10,0\%, respectivamente.

A leptina e a adiponectina foram dosadas por radioimunoensaio, no mesmo laboratório, no equipamento GAMA-C12, com kits que utilizam o método de duplo anticorpo PEG (Linco Research, St. Charles, MO, EUA), em soro adequadamente armazenado para tal. $\mathrm{O}$ kit para leptina utiliza leptina humana marcada com ${ }^{125}$ I e um antissoro de leptina humana, e o da adiponectina utiliza adiponectina de coelho marcada com ${ }^{125}$ I e um antissoro de coelho para adiponectina multi-espécie. Os intra-ensaio e inter-ensaio coeficientes de variação foram 3,4-8,3\% e 3,0-6,2\%, respectivamente.

Os dados coletados foram arquivados em planilhas do MS Excel versão 7 (MapInfo Corporation, Troy, NY, EUA). As análises estatísticas foram feitas no programa Epi Info, versão 7.1.5 (CDC, Atlanta, GA, EUA).

Foram calculadas, para cada grupo, médias e desvios padrões das seguintes variáveis contínuas com distribuição normal: idade, circunferência da cintura, glicose, colesterol, triglicerídeos, HDL-colesterol, LDL-colesterol, insulina, adiponectina e leptina. Para as variáveis contínuas de distribuições não normais, foram calculadas, para cada grupo, as medianas e desvios padrões das seguintes variáveis: HOMA-IR, pressão arterial sistólica (PAS) e pressão arterial diastólica (PAD). Para as variáveis categóricas, foram calculadas, por grupo, as frequências de SM e acantose nigricans. Foram calculadas, também por grupo, as frequências de aumento para: pressão arterial, circunferência da cintura, glicose e insulina. Calcularam-se ainda as frequências de cada grupo de alteração para: colesterol, triglicerídeos, HDL-colesterol e LDL-colesterol. Foram comparadas as médias das variáveis contínuas entre os grupos de obesos, com obeso grave, utilizando ANOVA, seguido de teste de Fisher para identificação dos grupos diferentes, com exceção da pressão arterial sistólica, para a qual foi utilizado na comparação das médias o teste de Kruskal Wallis seguido do teste de Mann-Whitney para a identificação dos grupos diferentes. Foram comparadas também as frequências de acantose nigricans, SM e das classificações aumento e alteração das variáveis categóricas entre os mesmos grupos, utilizando-se o qui-quadrado de Pearson, com exceção da hipertensão arterial sistêmica e glicose aumentada, os quais foram comparados pelo teste exato de Fisher.

Considerou-se significância estatística um valor de $\mathrm{p}$ menor que 0,05. Este estudo foi aprovado pelo Comitê de Ética e Pesquisa do Hospital Universitário Pedro Ernesto da Universidade do Estado do Rio de Janeiro, onde está cadastrado sob o número 173-CEP/ HUPE - CAAE; 0020.0.228.000-07.

\section{Resultados}

Das 82 crianças que participaram do estudo, 52 eram obesas graves e 32 eram obesas.

O grupo de obesos constituiu-se de 18 meninas (34,62\%) e 34 meninos (65,38\%), com média de idade de $97 \pm 17$ meses. Já o grupo de obesos graves, foi formado por 7 meninas $(21,88 \%)$ e 25 meninos $(78,13 \%)$, com média de idade de $94 \pm 20$ meses.

Não houve diferença estatisticamente significativa em relação às médias de idades e frequências dos gêneros ao se comparar os grupos com obesidade e com obesidade grave, assim, as comparações entre os grupos foram realizadas, sem estratificação por gênero ou idade.

As comparações entre os valores das médias ou medianas das variáveis contínuas clínicas e metabólicas entre os grupos com obesidade e obesidade grave se encontram na tabela 1.

As comparações das frequências de alterações de cada variável categórica clínica e metabólica entre os grupos estão na tabela 2 . 
Tabela 1. Comparações das médias das variáveis contínuas clínicas e metabólicas entre os grupos de crianças obesas e obesas graves.

\begin{tabular}{|c|c|c|c|}
\hline Variáveis & Obesos & Obesos graves & P valor \\
\hline Participantes(N) & $52(61,9 \%)$ & $32(31,1 \%)$ & \\
\hline Idade em meses & $97 \pm 17$ & $94 \pm 20$ & 0,56 \\
\hline \multicolumn{4}{|l|}{ Sexo } \\
\hline Feminino & $18(72 \%)$ & $7(28 \%)$ & 0,32 \\
\hline Masculino & $34(57,63 \%)$ & $25(42,37 \%)$ & 0,32 \\
\hline Circunferência da cintura $(\mathrm{cm})^{*}$ & $71,1 \pm 7,66$ & $81,23 \pm 9,31$ & 0.00001 \\
\hline Glicose (mg/dL) & $86,98 \pm 9,06$ & $87,03 \pm 8,02$ & 0,98 \\
\hline${ }^{1}$ PAS $(\mathrm{mmHg}) *$ & $90(92,5-102,5)$ & $100(90-110)$ & 0,007 \\
\hline${ }^{2} \mathrm{PAD}(\mathrm{mmHg})^{*}$ & $57,11 \pm 9,41$ & $62,96 \pm 11,97$ & 0,01 \\
\hline Colesterol total (mg/dL) & $169,75 \pm 33,83$ & $167,6 \pm 31,98$ & 0,72 \\
\hline${ }^{3} \mathrm{LDL}-\mathrm{C}(\mathrm{mg} / \mathrm{dL})$ & $106,59 \pm 31,13$ & $105,78 \pm 29,48$ & 0,9 \\
\hline${ }^{4} \mathrm{HDL}-\mathrm{C}(\mathrm{mg} / \mathrm{dL})$ & $43,36 \pm 9,29$ & $40,06 \pm 8,51$ & 0,1 \\
\hline Triglicerídeos (mg/dL) & $97,92 \pm 47,05$ & $106,03 \pm 48,61$ & 0,45 \\
\hline Insulina $(\mu \mid \mathrm{U} / \mathrm{mL})^{*}$ & $8,78 \pm 5,78$ & $11,70 \pm 6,26$ & 0,02 \\
\hline${ }^{5} \mathrm{HOMA}-\mathrm{IR}^{*}$ & $1,89 \pm 1,28$ & $2,55 \pm 1,48$ & 0,03 \\
\hline Adiponectina $(\mu \mathrm{g} / \mathrm{mL})$ & $13,25 \pm 5,27$ & $11,89 \pm 6,15$ & 0,28 \\
\hline Leptina (ng/mL) & $26 \pm 15,15$ & $24,45 \pm 20,36$ & 0,69 \\
\hline
\end{tabular}

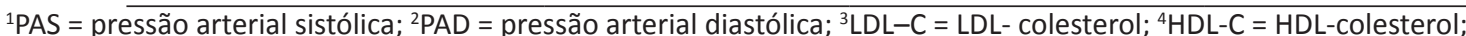
${ }^{5} \mathrm{HOMA}-\mathrm{IR}=$ homeostasis model assessment for insulin resistance.

* Médias que apresentam diferença estatística em relação às outras.

Tabela 2. Comparações das frequências de alterações de cada variável categórica clínica e metabólica, entre os grupos de crianças obesas e obesas graves.

\begin{tabular}{|l|c|c|c|}
\hline Variáveis & Obesos & Obesos graves & P valor \\
\hline Cintura $^{1}$ & $28(53,85 \%)$ & $52(100 \%)$ & $<0,001$ \\
\hline${\text { Pressão } \text { arterial }^{1}}^{\text {Acantose nigricans }}{ }^{2}$ & $0(0,0 \%)$ & $3(9,38 \%)$ & 0,052 \\
\hline Glicemia $^{3}$ & $13(25 \%)$ & $20(62,5 \%)$ & 0,001 \\
\hline Colesterol total $^{3}$ & $2(3,85 \%)$ & $1(3,13 \%)$ & 0,66 \\
\hline HDL-colesterol $^{4}$ & $25(48,08 \%)$ & $14(43,75 \%)$ & 0,87 \\
\hline LDL-colesterol $^{3}$ & $29(55,7 \%)$ & $25(78,13 \%)$ & 0,06 \\
\hline Triglicerídeos $^{3}$ & $8(15,38 \%)$ & $7(21,88 \%)$ & 0,64 \\
\hline Insulina $^{1}$ & $13(25 \%)$ & $10(31,25 \%)$ & 0,71 \\
\hline Síndrome Metabólica & & 0,004 \\
\hline
\end{tabular}

${ }^{1}$ Aumentada.

${ }^{2}$ Presente.

${ }^{3}$ Alterado $=$ limítrofe + aumentado.

${ }^{4}$ Diminuído. 


\section{Artigo original}

\section{Discussão}

O grupo estudado foi de crianças em idade tenra, isso explica o fato de algumas alterações metabólicas ainda não estarem tão explícitas. Entretanto, o achado de fatores de risco para doença cardiovascular aterosclerótica, como circunferência da cintura aumentada, HDL-colesterol baixo e triglicérides altos, em ambos os grupos, é preocupante. Além disso, achados como, circunferência da cintura, PAS, PAD, insulina e HOMA-IR, se mostram estatisticamente diferentes quando se comparam ambos os grupos, mostrando que a obesidade grave já demonstra consequências mais severas, até mesmo na idade tenra.

Ao comparar as frequências de circunferência da cintura aumentada entre os grupos, houve diferenças estatísticas e todos os integrantes do grupo de obesos graves apresentaram aumento da cintura, assim, esse grupo obteve maior frequência. Isso demonstra a importância dessa medida como indicadora de obesidade em crianças, conforme já mencionado na literatura. ${ }^{15}$

Na comparação das frequências de acantose entre os grupos, também houve diferença estatisticamente significativa, e pode-se observar uma prevalência superior no grupo de obesos graves. Isso pode demonstrar que crianças com obesidade grave já apresentam uma resistência insulínica mais acentuada, já que a acantose nigricans é um marcador de tal resistência, mesmo em crianças. ${ }^{21} \mathrm{E}$, de fato, as médias de HOMA-IR e as frequências de insulina aumentada são maiores nesse grupo, o que reforça o achado da resistência insulínica ser mais acentuada em obesos graves. É importante destacar que essas duas variáveis, média de HOMA-IR e frequência de insulina aumentada, também se apresentaram com diferença estatisticamente significativa, estando desfavoráveis no grupo de obesos graves.

Não houve diferença estatística nas médias de colesterol, LDL-colesterol e triglicerídeos entre os grupos estudados. Entretanto, as médias dessas variáveis, em ambos os grupos, se situaram acima do ponto de corte recomendado para a faixa de normalidade para crianças no Brasil. ${ }^{19}$ Desse modo, apesar de não haver diferença entre os grupos, já é possível observar o surgimento destes fatores de risco para doenças cardiovasculares até mesmo nos obesos não classificados como graves. As prevalências de colesterol alterado também foram importantes, apesar de não haver diferença estatística entre os grupos estudados.

Quanto às médias de HDL-colesterol não houve diferença estatística significativa, mas é possível iden- tificar que essas médias, em ambos os grupos, estão abaixo do ponto de corte, para crianças, recomendado como normal, no Brasil. ${ }^{19} \mathrm{O}$ mesmo se observa quando se compara a prevalência de HDL-colesterol alterado: não há diferença estatisticamente significativa, entre os grupos estudados, mas existem prevalências elevadas em ambos os grupos. Esse fato reforça a preocupação em relação à obesidade como fator de risco para aterogênese, até mesmo em crianças obesas que não tenham a classificação de obesidade grave. Isso porque já foi demonstrado na literatura, desde longa data, que a fração HDL-colesterol é antiaterogênica. ${ }^{22}$

Em relação à prevalência da SM, não houve diferença estatística entre os grupos. Por outro lado, pode-se observar uma prevalência considerável nos dois grupos. É importante ressaltar que muito vem sendo debatido em relação a esta variável, uma vez que, na literatura, não há um consenso entre os critérios diagnósticos. ${ }^{23}$ Uma proposta seria a utilização da resistência insulínica, representada pelo HOMA-IR, no lugar de glicemia de jejum, ${ }^{24}$ visto que crianças em idade tenra, embora apresentem alterações no metabolismo dos carboidratos, como visto nos resultados aqui apresentados, ainda não apresentam DM2 nem glicemia de jejum alterada em prevalência considerável.

A leptina é um hormônio que tem papel na fisiopatologia da obesidade e SM. Esse hormônio pode contribuir para a resistência à insulina e parece ter um efeito pró-trombótico, atuando junto com os ácidos graxos livres e a insulina para estimular a atividade simpática e vasoconstrição. ${ }^{25}$ Desse modo, a insulina e leptina interagem regulando a função vascular, podendo gerar consequências na disfunção vascular da SM. Não há um consenso na literatura a respeito do ponto de corte em crianças. No entanto, um estudo do presente grupo de pesquisadores, realizado na mesma população, ${ }^{26}$ mostrou que se for adotado o ponto de corte de $13,4 \mathrm{ng} /$ $\mathrm{ml}$ existe uma sensibilidade e uma especificidade de $68 \%$ e $69 \%$, respectivamente, para a SM. Assim, usando esse ponto de corte, observa-se que todas as crianças de ambos os grupos estão acima, apesar de não haver diferença estaticamente significativa entre tais grupos.

Os valores das concentrações de adiponectina não tiveram diferença estatística entre os grupos. Apesar disso, é importante comentar que outros estudos mostram que os baixos níveis séricos de adiponectina se correlacionam com a dislipidemia. ${ }^{27}$ Logo, é possível dizer que adiponectina exerce um papel antiaterogênico, por meio da sua influência no metabolismo lipídico.

Em relação à hipertensão arterial, não se observou 
uma diferença estatisticamente significativa, nem uma prevalência muito significativa. Isso pode ser explicado pela idade tenra, já que são crianças impúberes e com poucas alterações metabólicas. Entretanto, se observam diferenças estatísticas ao analisar as mediana de PAS e médias de PAD, mostrando que ambas estão precocemente mais aumentadas no grupo de obesos grave, demonstrando, assim, um maior risco para hipertensão arterial nesse grupo. A maior prevalência de insulina aumentada no grupo, possivelmente, contribui para aquele achado, visto que a insulina aumenta a reabsorção de sódio renal. ${ }^{28}$

A partir dessa perspectiva, é possível afirmar que os resultados do presente estudo mostraram que crianças com obesidade grave apresentam algumas alterações metabólicas mais intensas em relação a crianças com obesidade não grave. Apesar disso, ainda há necessidade de mais trabalhos para a melhor identificação e caracterização dos distintos comportamentos genéticos, fisiopatológicos e os fatores ambientais associados com a maior gravidade. Assim, será possível pensar em novas abordagens de prevenção e tratamento.

\section{Agradecimento}

À Isabel Rey Madeira, minha orientadora, pela oportunidade de participar da pesquisa e pelo aprendizado que pode me proporcionar.

À Fernanda Mussi Gazolla, pelo companheirismo durante o ambulatório e pelo auxílio na análise dos dados estatísticos.

\section{Referências}

1. Ng M, Fleming T, Robinson M, et al. Global, regional, and national prevalence of overweight and obesity in children and adults during 1980-2013: a systematic analysis for the Global Burden of Disease Study 2013. Lancet.2014;384:766-81

2. Brasil. IBGE. Pesquisa de orçamentos familiares - POF 20022003.

3. Sun SS, Liang R, Huang TT, et al. Childhood obesity predicts adult metabolic syndrome: the Fels Longitudinal Study. J Pediatr. 2008;152:191-200.

4. Juhola J, Magnussen CG, Viikari JS, et al. Tracking of serum lipid levels, blood pressure, and body mass index from childhood to adulthood: the Cardiovascular Risk in Young Finns Study. J Pediatr. 2011;159:584-90.

5. Zimmet P, Alberti G, Kaufman F, et al. The metabolic syndrome in children and adolescents. Lancet. 2007;369:2059-61.

6. Fonseca-Alaniz MH, Takada J, Alonso-Vale MI, et al. Adipose tissue as an endocrine organ: from theory to practice. J Pediatr (Rio J). 2007;83:203.

7. Ten S, MacLaren N. Insulin resistance syndrome in children. J ClinEndocrinol Metab. 2004;89:2526-39.

8. Matthews DR, Hosker JP, Rudenski AS, et al. Homeostasis model assessment: insulin resistance and B-cell function from fasting plasma glucose and insulin concentrations in man.
Diabetologia. 1985;28:412-9.

9. Yamazaki $\mathrm{H}$, Ito $\mathrm{S}$, Yoshida $\mathrm{H}$. Acanthosis nigricans is a reliable cutaneous marker of insulin resistance in obese Japanese children. Pediatr Int. 2003;45:701-5.

10. Skelton JA, Cook SR, Auinger P, et al. Prevalence and trends of severe obesity among US children and adolescents. AcadPediatr. 2009;9:322-9.

11. Freedman DS, Mei Z, Srinivasan SR, et al. Cardiovascular risk factors and excess adiposity among overweight children and adolescents: the Bogalusa Heart Study. J Pediatr. 2007;150:12.

12. Baker JL, Olsen LW, Sørensen TI. Childhood body-mass índex and the risk of coronary heart disease in adulthood. $\mathrm{N}$ Engl $\mathrm{J}$ Med. 2007;357:2329-37.

13. Tirosh A, Shai I, Afek A, et al. Adolescent BMl trajectory and risk of diabetes versus coronary disease. $\mathrm{N}$ Engl J Med. 2011;364:1315-25.

14. Marshall WA, Tanner JM. Variations in the pattern of pubertal changes in girls. Arch Dis Child. 1969;44:291-303.

15. de Onis M, Onyango AW, Borghi E, et al. Development of a WHO growth reference for school-aged children and adolescents. Bull of the World Health Organization. 2007;85:660-7.

16. Fleiss JL, Levin BA, Levin B, et al. Statistical methods for rates and proportions. 3rd ed. Oxford: Wiley-InterScience; 2003.

17. Burke JP, Hale DE, Hazuda HP, et al. A quantitative scale of acanthosis nigricans. Diabetes Care. 1999;22:1655-9.

18. Fernández JR, Redden DT, Pietrobelli A, et al. Waist circumference percentiles in nationally representative samplesof African-American, European American, and Mexican-American children and adolescents. J Pediatr. 2004;145:439-44.

19. Sociedade Brasileira de Cardiologia. I Diretriz de Prevenção da Aterosclerose na Infância e na Adolescência. Arq Bras Cardiol. 2005;85.

20. Friedewald WT, Levy RI, Fredrickson DS. Estimation of the concentration of low-density lipoprotein cholesterol in plasma without use of preparative ultracentrifugate. Clin Chem. 1972;18:499-502.

21. Miura N, Ikezaki A, Iwama S, et al. Genetic factors and clinical signifi cance of acanthosis nigricans in obese japanese children and adolescents. Acta Paediatr. 2006;95:170-5.

22. Reaven GM. Role of insulin resistance in human disease. Diabetes. 1988;37:1595-607.

23. Reinehr T, de Sousa G, Toschke AM, et al. Comparison of metabolic syndrome prevalence using eight different definitions: a critical approach. Arch Dis Child. 2007;92:1067-72.

24. Cavali M de L, Escrivão MA, Brasileiro RS, et al. Metabolic syndrome: comparison of diagnosis criteria. J Pediatr (Rio J). 2010;86:325-30.

25. Konstantinides K, Schafer K, Neels JG, et al. Inhibition of endogenous leptin protects mice from arterial and venous thrombosis. Artherioscler Thromb Vasc Biol. 2004;24:2196-201.

26. Madeira IR, Gazolla F, Collett-Solberg P, et al. Leptin as a predictor of metabolic syndrome in prepubertal children. archives of endocrinology and metabolism. 2016: Aceito em fevereiro para publicação na revista Archives of Endocrinology and Metabolism.

27. Pilz S, Horejsi R, Möller R, et al. Early atherosclerosis in obese juveniles is associated with low serum levels of adiponectin. $J$ Clin Endocrinol Metab. 2005;90:4792-6.

28. Steinberger J, Daniels SR. Obesity, insulin resistance, diabetes, and cardiovascular risk in children: an American Heart Association scientific statement from the Atherosclerosis, Hypertension, and Obesity in the Young Committee (Council on Cardiovascular Disease in the Young) and the Diabetes Committee (Council on Nutrition, Physical Activity, and Metabolism). Circulation. 2003;107:1448-53. 